\title{
Inadequate magnetic resonance imaging decision pattern in case of suspicious prostate alterations and the advantage of ultrasound target biopsies
}

\author{
Lunacek $A^{1 *}$, Stiassny $F^{2}$, Oeser $R^{3}$, Pelzer $A^{4}$, Bektic $J^{5}$, Horninger $W^{5}$, Shariat $S^{6}$ and Plas $E^{1}$ \\ ${ }^{1}$ Department of Urology, Hanusch Krankenhaus, Vienna, Austria \\ ${ }^{2}$ Radiologicum Penzing, Vienna, Austria \\ ${ }^{3}$ Oeser GmbH, Vienna, Austria \\ ${ }^{4}$ Department of Urology, Hospital Wels-Grieskirchen, Wels, Austria \\ ${ }^{5}$ Department of Urology, Medical University of Innsbruck Austria \\ ${ }^{6}$ Department of Urology, Medical University of Vienna Austria
}

\begin{abstract}
Introduction: To evaluate and compare the benefits of multiparametric magnetic resonance imaging ( $\mathrm{mpMRI}$ ) in combination with fusion prostate biopsy (fPBx) and find out what happened with patients underwent single MRI without fPBx.

Materials and methods: 125 patients underwent prostate mpMRI. Sixty-five of these patients additionally underwent ultrasound-guided fPBx. Prostate lesions were scored using the Prostate Imaging Reporting and Data System version 2, and the results were verified with prostate biopsies.

Results: Sixty-five patients (52\%) underwent fPBx after mpMRI, and 60 patients (48\%) underwent mpMRI alone as ordered by their physician. Of the 65 men who underwent $\mathrm{fPBx}$, PCa was verified in 33 men (50.77\%) who exhibited suspicious lesions on mpMRI, whereas 20 men (30.77\%) yielded negative results, although suspicious lesions were observed by mpMRI. Among the 65 men, 7 (10.76\%) and 5 (7.7\%) men had atypical small acinar proliferation and chronic prostatitis, respectively. The mean PCa detection rate for standard biopsy was 21.9\% (SD, 26.4), whereas that of the mpMRI combined with fPBx was 49.5\% (SD, 41.9) (p-value $<0.0001)$

Of the 53 patients who underwent biopsy, 13 patients underwent the algorithm mpMRI/fPBx for first-line diagnosis, with nearly $70 \%$ patients ( $\mathrm{n}=9$ ) experiencing positive results.

Conclusion: $\mathrm{fPBx}$ detected considerably more cases of $\mathrm{PCa}$ than standard biopsies, demonstrating the clear advantages of the former method. Because of the wellknown low specificity of mpMRI, this method alone is insufficient for the diagnosis of PCa if no further diagnostic interventions are planned.
\end{abstract}

\section{Introduction}

Prostate cancer $(\mathrm{PCa})$ is the most common cancer among the European population [1]. Prostate biopsies are recommended to verify PCa in patients with elevated prostate-specific antigen (PSA) levels and/or suspicious digital rectal exams (DREs) or Prostate Imaging Reporting and Data System (PIRADS) scores $\square 3$ upon mpMRI (European Association of Urology [EAU] Guidelines). The role of biopsies has changed since their introduction; in addition to cancer detection, biopsies are used for making clinical decisions regarding therapeutic planning and as follow-up tests for active surveillance. Data about histology, prostate specific antigen density, tumor location and extent are essential for accurate risk assessment regarding the biology and clinical aggressiveness of the encountered tumors [2-4].

The 10-12-core transrectal ultrasound-guided systematic mapping biopsy (TRUS-GB) is still the primary diagnostic modality for the detection of $\mathrm{PCa}[1,5]$; however, this approach has a significant falsenegative rate of up to $47 \%$, with up to $38 \%$ of patients undergoing a repeat prostate biopsy within 5 years [6-8].
The current index and extent of cancer is often underestimated, leading to a high rate of cancer upgrading and upstaging [9-11]. mpMRI as a single diagnostic tool for detecting PCa has limitations. The sensitivity (up to 93\%) is high, but this can be compromised by motion artifacts, lack of standardized acquisition, analysis methods and lack of experience, leading to variabilities in the results. The specificity of $41 \%$ is low, and common signal alterations include postbiopsy changes, hyperplastic nodules and inflammation.

Hence, mpMRI alone is not recommended for the detection of $\mathrm{PCa}$ and should not be used as a single examination for patients having prostate or PSA alterations although imaging and nuclear medical techniques become fundamental in the detection of prostate cancer [12-15].

${ }^{*}$ Correspondence to: Lunacek A, Department of Urology, Hanusch Krankenhaus, Heinrich Collin Strasse, Vienna, Austria, Tel: 00439102186011 ; Fax: 004391021 86019; E-mail: andreas.lunacek@acmp.at

Received: October 21, 2019; Accepted: October 28, 2019; Published: October 30, 2019 
We retrospectively evaluated the advantages of mpMRI combined with $\mathrm{PPBx}$ and distinguished inadequate decisions resulting from a single mpMRI.

\section{Patients and methods}

\section{Patients}

We performed a single-center retrospective study at the Department of Urology and the Department of Radiology of Hanusch Krankenhaus, Vienna. Data of 125 men were retrospectively collected from November 2016 to November 2017. The patients were classified into five groups, of which four (i.e., PCa, negative biopsy, atypical small acinar proliferation [ASAP], and chronic prostatitis) underwent both mpMRI and fPBx. The inclusion criteria were having at least one prior negative prostate biopsy with a suspicion of PCa (i.e., abnormal DRE, elevated PSA levels or PSA velocity) and findings of ASAP or high-grade prostatic intraepithelial neoplasia (HGPIN) in at least four biopsy samples further selected cases of young patients in excellent physical conditions.

The collected data included age, PSA level, PIRADS version 2, Gleason Score (GS), number of positive standard biopsy and mpMRI plus US-guided $\mathrm{fPBx}$ (combined biopsy), and tumor volume.

In total, 60 patients underwent mpMRI alone, as recommended by their treating physician, after referral to the radiologic department following the diagnosis of elevated PSA levels or suspected PCa; however, they were lost to follow-up after obtaining their radiological report. These data were documented using Microsoft ${ }^{\odot}$ Excel (Microsoft Corp, Redmond, WA, USA) and then transferred to NCSS 10 Statistical Software 2015 version (NCSS, LLC., Kaysville, UT, USA) for statistical analysis. STATISTICA version 12 (Statsoft, Palo Alto, CA, USA) was also used for the statistical analysis.

The study was planned according to the guidelines in the synthesis of qualitative research (ENTREQ) on equator-network.org [16]. All procedures followed were in accordance with the ethical standards of the responsible committee on human experimentation and with the Helsinki Declaration of 1975, as revised in 2008. For this study, formal consent of patients was not required.

\section{Multiparametric magnetic resonance imaging}

All patients underwent mpMRI performed on a 1.5-Tesla MR scanner (Philips Achieva Version 2 Series, Phillips, Amsterdam, The Netherlands) using a multichannel external phase array body coil, including T2-weighted imaging (T2WI) dynamic contrastenhanced and diffusion-weighted imaging (DWI). The imaging protocol of PIRADS version 2 was followed according to the following recommendations of the American College of Radiology (ACR): reference slice thickness, $3 \mathrm{~mm}$ and field of view, $160 \mathrm{~mm}$ for DWI and $180 \mathrm{~mm}$ for T2WI in three planes. Further, we used four B values $(0,100$, 1000 , and $1500 \mathrm{~s} / \mathrm{mm}^{2}$ ) for interpreting DWI. The results were analyzed and classified by experienced uroradiologists. DWI and T2WI were used for analyzing the peripheral zone (PZ) and transitional zone (TZ), respectively, and notable regions of interest (ROIs) were given an MRI suspicion score according to the PIRADS 2 scoring system following the ACR/European Society of Urogenital Radiology recommendations [17]. The lesions detected in the TZ were assessed in axial, sagittal, and coronal planes, while those detected in the $\mathrm{PZ}$ were assessed only in the axial plane. The lesions were scored from 1 to 5 , with 1 indicating the lowest suspicion of PCa. The TZ in the T2WI sequence was classified as PIRADS 1 if homogeneous intermediate signal intensity/normal was noted; PIRADS 2, circumscribed hypointense or heterogeneous encapsulated nodules/benign prostatic hyperplasia; PIRADS 3, heterogeneous signal intensity with obscured margins and also includes others that do not qualify as PIRADS 2, 4, or 5; PIRADS 4, lenticular or non-circumscribed, homogeneous, moderately hypointense and $<1.5$ $\mathrm{cm}$ in the greatest dimension; and PIRADS 5, same as 4 except with $\geq 1.5 \mathrm{~cm}$ in the greatest dimension or definite extraprostatic extension/ invasive behavior. The PZ in the DWI sequence was classified as PIRADS 1 if no abnormality on apparent diffusion coefficient (ADC) and a high b-value on DWI were noted; PIRADS 2, for indistinct hypointensity on ADC; PIRADS 3, for focal mildly/moderately hypointense on ADC and isointense/mildly hyperintense on high b-value DWI); PIRADS 4, for focal markedly hypointense on ADC and markedly hyperintense on high b-value DWI and with $<1.5 \mathrm{~cm}$ in the greatest dimension; PIRADS 5 , the same as 4 but with $\geq 1.5 \mathrm{~cm}$ in the greatest dimension or definite extraprostatic extension/invasive behavior. The images were saved on a $\mathrm{CD}$ for transfer to the ultrasound system [18] (Figure 1a).

\section{Ultrasound-guided fPBx}

The MR images were entered into the ultrasound system (Aplio 500 Toshiba). The patients were prepared for MRI according to our department's specific protocol [19]. T2W MRI sequences were fused to the real-time ultrasound. The fusion of the methods is provided on electromagnetic position sensors that allow the registration of simultaneous movements of the ultrasound and MRI pictures.

Biopsies were performed under antibiotic prophylaxis dependent on the results of rectal swab, and $93 \%$ of patients received flourochinolons. For patients with grade $\geq 2$ lesions on MRI, 3-5 targeted biopsy specimens were first obtained from the described and marked ROIs (Figure 1a and 1b.) and 12-core systematic biopsies were then performed independent of MRI results in the axial plane. The protocol entails that samples encompassing the bilateral anterior, apex, mid, base, and lateral locations be obtained [14]. A spring-loaded gun (18-Gauge needle) was used, and biopsies were performed using an end-fire transrectal probe (Toshiba 11c3) by a single urologist supported by two uroradiologists. The patients were in the lateral position and received local anesthesia according to the department's protocol with a lidocaine suppository plus a periprostatic nerve block [15]. mpMRI and $\mathrm{fPBx}$ were performed with a 1-5-week interval. The specimens were graded based on the International Society of Urological Pathology and World Health Organization guidelines.

\section{Result}

The demographics are presented in Table 1. One hundred and twenty-five patients underwent mpMRI. The mean age was 64 (standard deviation $[\mathrm{SD}], 10$ ) years, and patients diagnosed with PCa had an average age of 68 (SD, 10) years. Sixty-five patients underwent $\mathrm{PPBx}$, of whom 33 patients were verified to have PCa via both biopsy techniques. Twenty patients had negative results, although suspicious lesions were noted by mpMRI. Of the cohorts with positive and negative biopsy results $(n=53), 13$ patients $(24.52 \%)$ underwent the combined biopsy technique for first-line diagnosis, of whom 9 patients $(69.2 \%)$ were verified to have PCa and 4 patients (30.8\%) were not.

Sixty patients underwent mpMRI alone. We tried to contact these patients who underwent mpMRI only to determine what was done after MRI.

The mean tumor diameter by MRI was 10.7 (SD, 5.2) $\mathrm{mm}$, and the mean volume of PCa was 45.9 (SD, 1.3) cc, while that of the normal prostate (i.e., negative biopsies, ASAP, and chronic prostatitis) was 48.2 (SD, 17.8) cc. The mean PSA level of the patient population was 9.81 


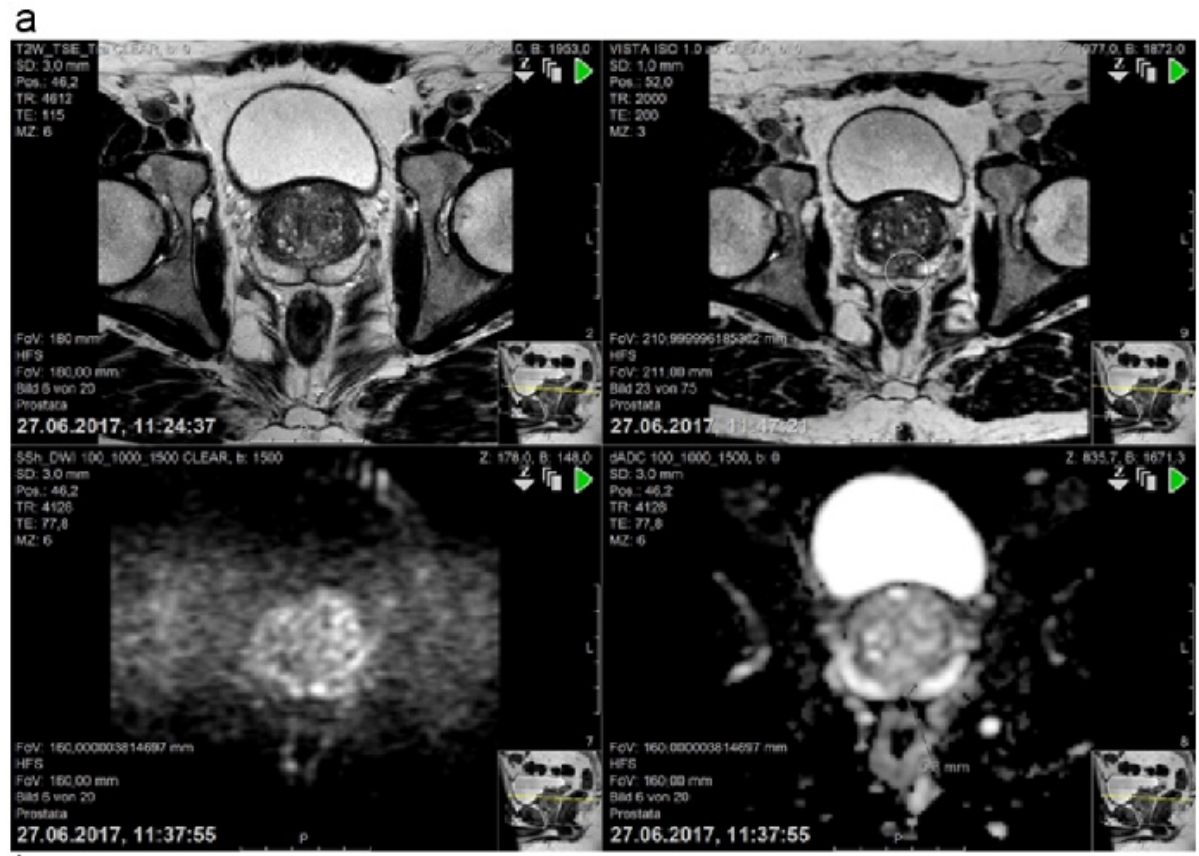

b

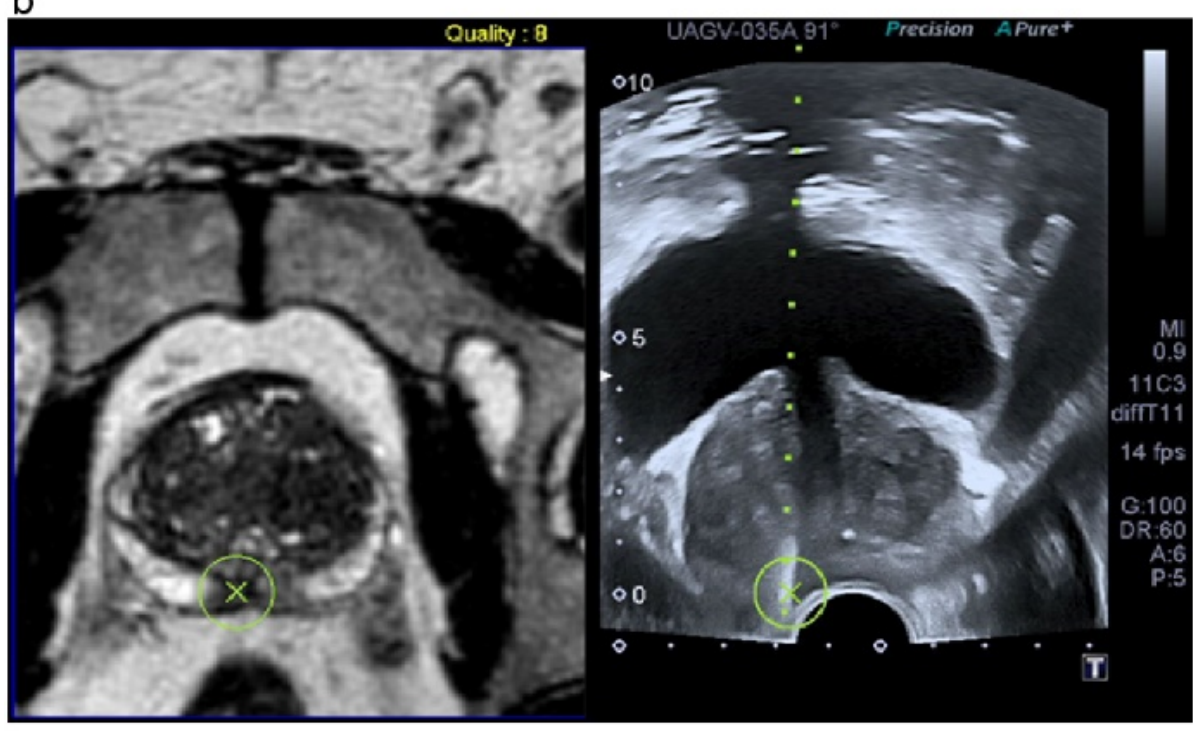

Figure 1. a) History of DWI b:0 (top right) where a suspicious area could be found; DWI b:1500 (top left) presents a typical hyperintense area right in the peripheral zone and the corresponding hypointense area in ADC (bottom right), which established the diagnosis of PIRADS 4. T2W image (bottom left) shows a possible lesion that is verified by DWI and ADC. b) The fusion of suspicious areas in MRI and the corresponding ultrasound with the region of interest. The result proves the MRI suspicion, Gleason score $8(4+4)$.

Abbreviations: ADC, apparent diffusion coefficient; DWI, diffusion-weighted imaging; MRI, magnetic resonance imaging; PIRADS, Prostate Imaging Reporting and Data System

Table 1. Patient demographics

\begin{tabular}{|c|c|c|c|c|c|c|c|c|c|c|c|}
\hline Group & Patient [\#] & $\begin{array}{c}\text { Distribution } \\
{[\%]}\end{array}$ & $\begin{array}{l}\text { Mean age }[y] \\
\text { (SD) }\end{array}$ & $\begin{array}{c}\text { Mean PSA } \\
{[\mathrm{ng} / \mathrm{ml}] \text { (SD) }}\end{array}$ & $\begin{array}{c}\text { Mean Size } \\
\text { TU MRI } \\
\text { [mm] (SD) }\end{array}$ & $\begin{array}{c}\text { Mean P-Vol } \\
\text { [g] (SD) }\end{array}$ & $\begin{array}{c}\text { Mean } \\
\text { PIRADS } \\
\text { (SD) }\end{array}$ & $\begin{array}{l}\text { Mean } \\
\text { Gleason- } \\
\text { Score } \\
\text { Standard }\end{array}$ & $\begin{array}{c}\text { Mean } \\
\text { Gleanson- } \\
\text { Score Fusion }\end{array}$ & $\begin{array}{c}\text { Mean } \\
\text { Standard } \\
\text { Deduction } \\
\text { [\%] (SD) }\end{array}$ & $\begin{array}{c}\text { Mean Fusion } \\
\text { Deduction } \\
\text { [\%] (SD) }\end{array}$ \\
\hline Pca & 33 & 26.4 & $68(10)$ & $15.5(24)$ & $10(5)$ & $48.2(17.8)$ & $3.8(0.9)$ & $6.9(1.0)$ & $7.1(0.9)$ & $24.5(27.8)$ & $56.2(40.7)$ \\
\hline eg. BX & 20 & 16 & $64(9)$ & $7.6(4.7)$ & $13(6)$ & $47.0(9.6)$ & $3.7(0.8)$ & & & & \\
\hline $\begin{array}{l}\text { MRI without } \\
\text { BX }\end{array}$ & 60 & 48 & $62(10)$ & $7.2(4.6)$ & & $43.60(10.9)$ & $2.72(1)$ & & & & \\
\hline ASAP & 7 & 5.6 & $62(9)$ & $9.1(3.8)$ & $8(4)$ & $48.7(7.8)$ & $3.5(0.8)$ & & & $8.3(5.3)$ & $5.0(11.2)$ \\
\hline $\begin{array}{c}\text { chron. } \\
\text { Prostatitis }\end{array}$ & 5 & 4 & $64(7)$ & $8.1(3.4)$ & $12(6)$ & $50.00(10.2)$ & $4.2(0.5)$ & & & & \\
\hline Total & 125 & $100.00 \%$ & $64(10)$ & $9.81(13.6)$ & $10.7(5.2)$ & $45.9(12.3)$ & $3.3(1.1)$ & & & $21.9(26.4)$ & $49.5(41.9)$ \\
\hline
\end{tabular}


(SD, 13.6) $\mathrm{ng} / \mathrm{ml}$. The mean PSA levels of patients with PCa, negative biopsy findings, ASAP, chronic prostatitis, and of those who only underwent mpMRI were 15.5 (SD, 24), 7.6 (SD, 4.7), 9.1 (SD, 3.8), 8.1 (SD, 3.4), and $7.2(\mathrm{SD}, 4.6) \mathrm{ng} / \mathrm{ml}$, respectively. The mean GS of patients examined using the standard/combined biopsy techniques was 6.9 (SD 1.0)/7.1 (SD, 0.9), and the mean PIRADS was 3.3.

The mean PCa detection rate for the standard was $21.9 \%$ (SD, 26.4), whereas that of the mpMRI combined with $\mathrm{fPBx}$ was $49.5 \%$ (SD, 41.9). The scatterplot (Figure 2) is shown as a function of the standard detection rate and the rate of the fusion biopsy per patient. The $\mathrm{x}$-axis indicates the $\mathrm{PPBx}$ detection rate, while the $\mathrm{y}$-axis indicates the detection rate of the standard protocol. The size of the spots represents the number of patients who had the same set of data. Figure 3 shows the data distribution as histograms. The standard protocol is represented as vertical lines, and the detection rate of $\mathrm{fPBx}$ as horizontal bar charts.

The histogram of the $\mathrm{PBBx}$ detection rate shows a U-distribution, whereas the detection rate of the standard protocol is crooked on the right side. As shown in Figure 4, the standard protocol yielded almost $100 \%$ of positive specimens. The greatest frequency detected with the standard biopsy techniques was $0 \%$ and $8 \%$. (Figure 4). Meanwhile, fPBx yielded a $100 \%$ detection rate in 11 patients. Twelve patients had negative $\mathrm{fPBx}$, but they had at least one positive biopsy on the standard biopsy technique.

The box plot (Figure 5) shows the detection rate of both cohorts. The median detection rate of the standard method is $12.5 \%$ (IRQ 0.25 ), while that of the combined method is $50 \%$ (IRQ: 1). This result means that the combination of the standard and combined biopsy techniques yielded at least $50 \%$ of the positive results. The Grading scores of the combined technique were higher (range, 7-9) than those of the standard biopsy (range, 6-9).

The standard technique had a detection rate of $24.5 \%$ (SD, 27.8), and the combined had a rate of $56.2 \%$ (SD, 40.7). For patients with
ASAP, the detection rate of the standard biopsy was $8.3 \%$ (SD, 5.3), and for the combined technique, the detection rate was $5 \%(\mathrm{SD}, 11.2)$.

Overall, 629 samples were obtained, of which 468 samples were analyzed using the standard biopsy technique. One hundred and one samples $(21.6 \%)$ were positive.

One hundred and sixty-one samples were analyzed using the combined technique, and 81 (50.3\%) were positive. The odds ratio was 3.679 .

Testing the proportion under $\mathrm{H}_{0:} \mathrm{P}_{1}=\mathrm{P}_{2}$ versus $\mathrm{H}_{1:} \mathrm{P}_{1} \otimes \mathrm{P}_{2}$ using the two-sided Wald chi-squared test (P1-P2) resulted in a significant p-value of $<0.0001$. The majority of the combined biopsy techniques resulted in the positive biopsy cohort having a PIRADS score of 4 and $5(\mathrm{n}=33,81.8 \%$; PIRADS 2: 5 [15.1\%]; PIRADS 3: 1 [3.1\%]; PIRADS 4: 20 [60.6\%], PIRADS 5: 7 [21.2\%]).

The majority of the "mpMRI alone" cohort $(n=60)$ had a PIRADS score of 2, but to emphasize the significance of mpMRI, $40 \%$ of the cohort had a PIRADS score $\mathbb{\nabla}$; therefore, $\mathrm{PPBx}$ is indicated (PIRADS 2: 36 [60\%], PIRADS 3: 7 [11.7\%], PIRADS 4: 14 [23.3\%], and PIRADS $5: 3[5 \%])$.

Using the standard biopsy technique, an average of $10 \%$ and a median of $8 \%$ of the group had PIRADS 2 , an average and a median of $42 \%$ of the group had PIRADS 3 , an average of $20 \%$ and a median of $13 \%$ of the group had PIRADS 4 , and an average of $36 \%$ and a median of $29 \%$ of the group had PIRADS 5.

In contrast, when using the combined technique, an average of $20 \%$ and a median of $0 \%$ of the group had PIRADS 2 , an average and a median of $50 \%$ of the group had PIRADS 3 , an average of $44 \%$ and a median of $33 \%$ of the group had PIRADS 4 , and an average of $90 \%$ and a median of $100 \%$ of the group had PIRADS 5 (Figure 6).

The cohort of the selected cases represent a significant result according to $(\mathrm{p}=0.000001)$ age. According to the PIRADS results, no significance was found ( $\mathrm{p}=0.952486)$.

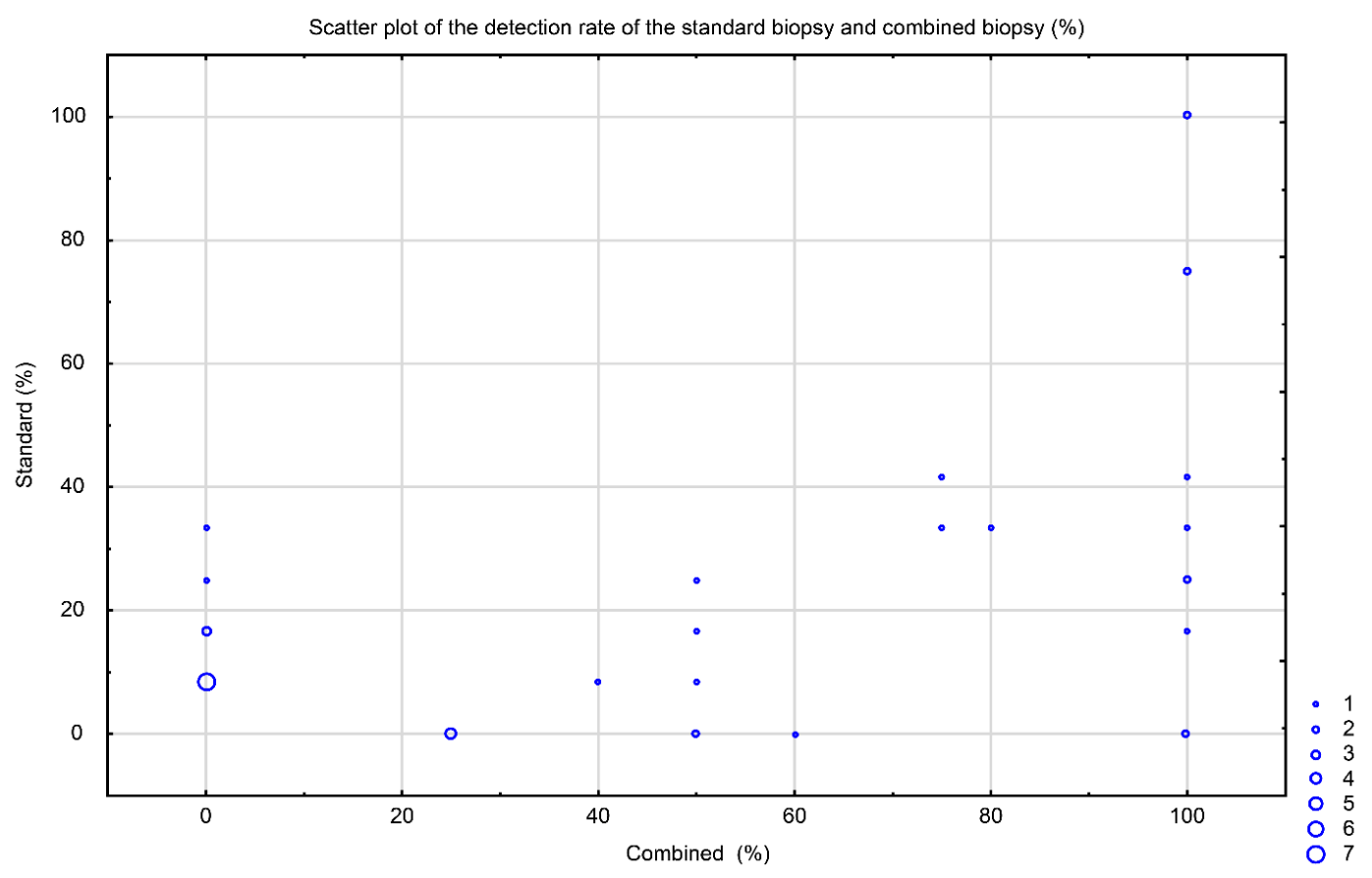

Figure 2. Scatterplot showing the detection rate of the standard biopsy and the combined biopsy techniques 


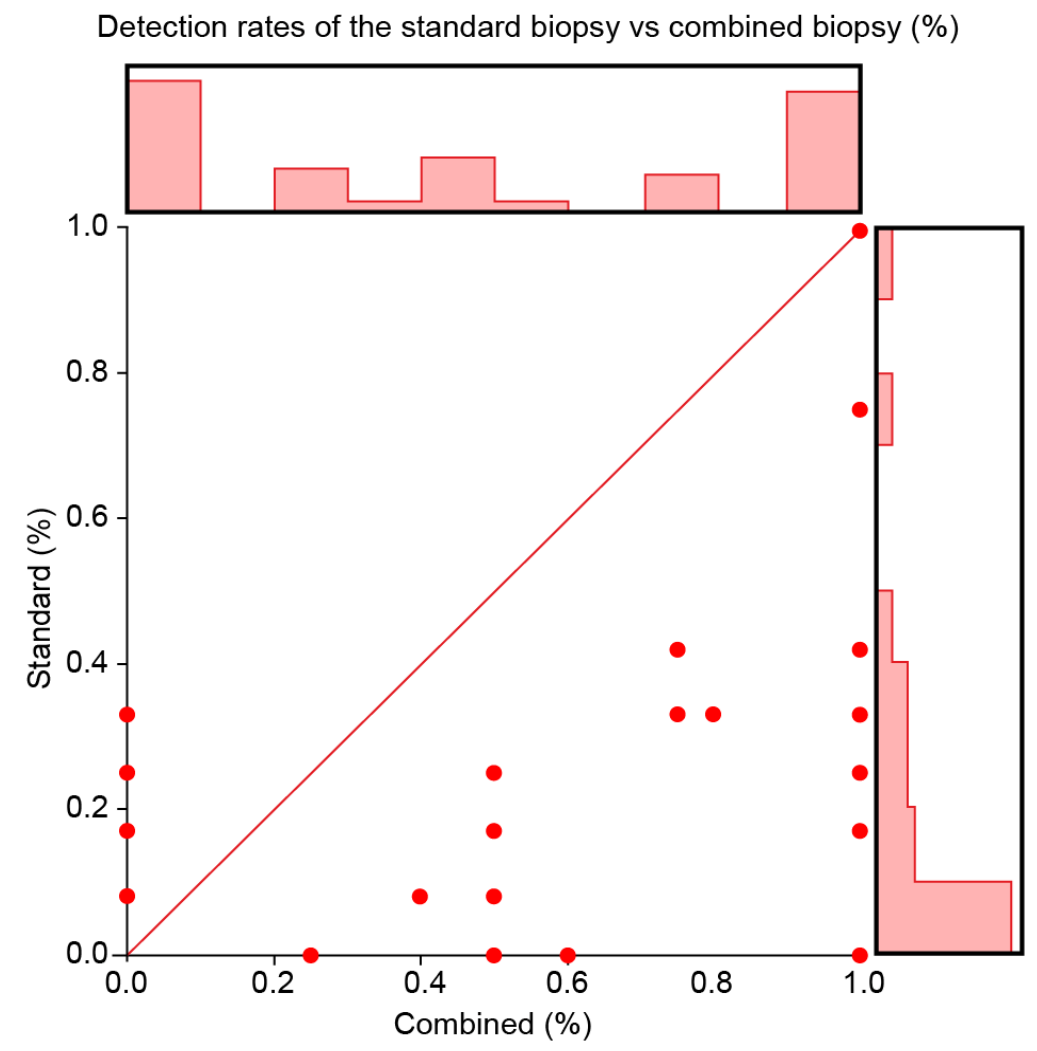

Figure 3. Data distribution in terms of histograms

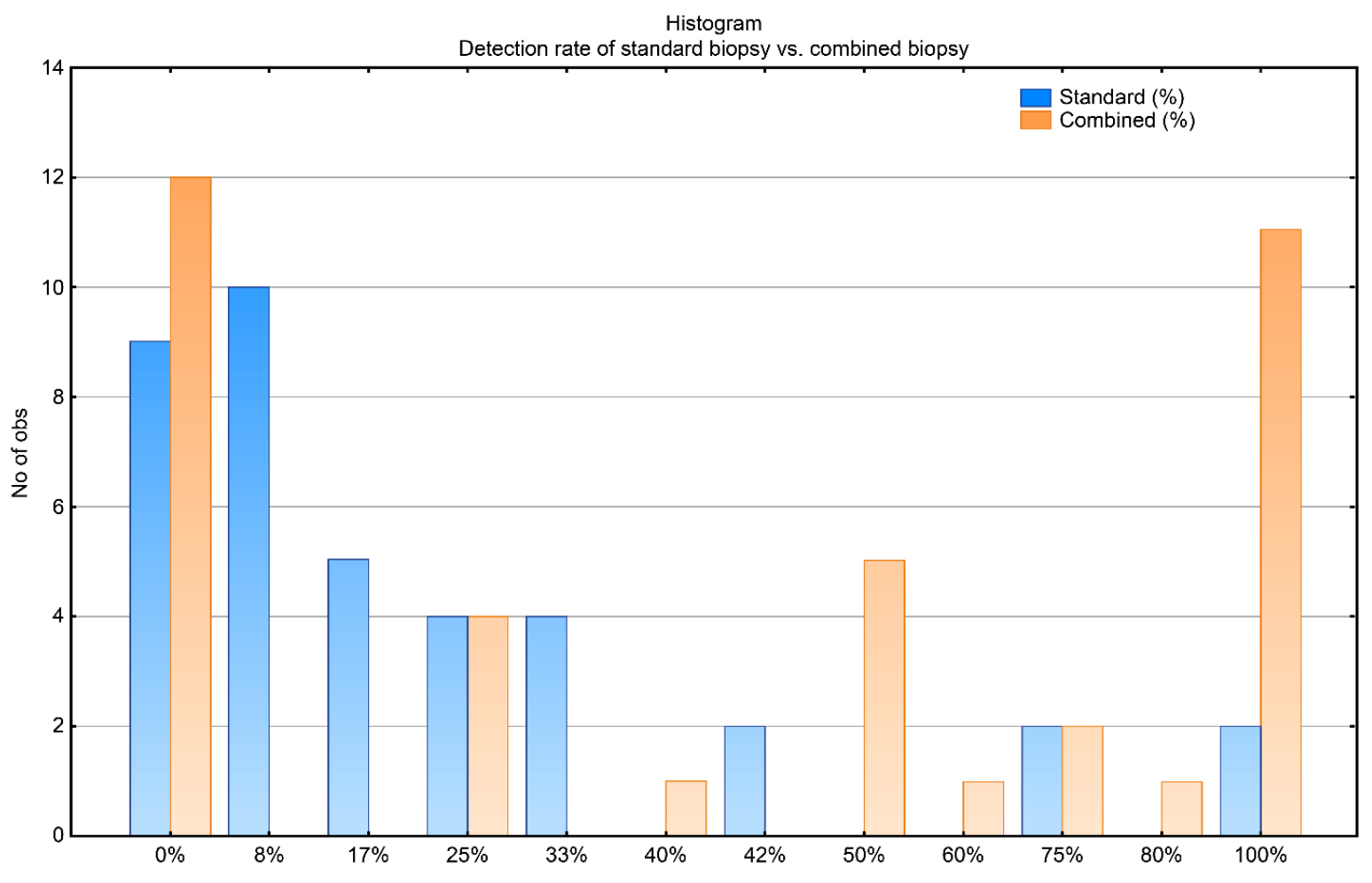

Figure 4. Comparison of the detection rate between the standard and combined biopsy techniques 


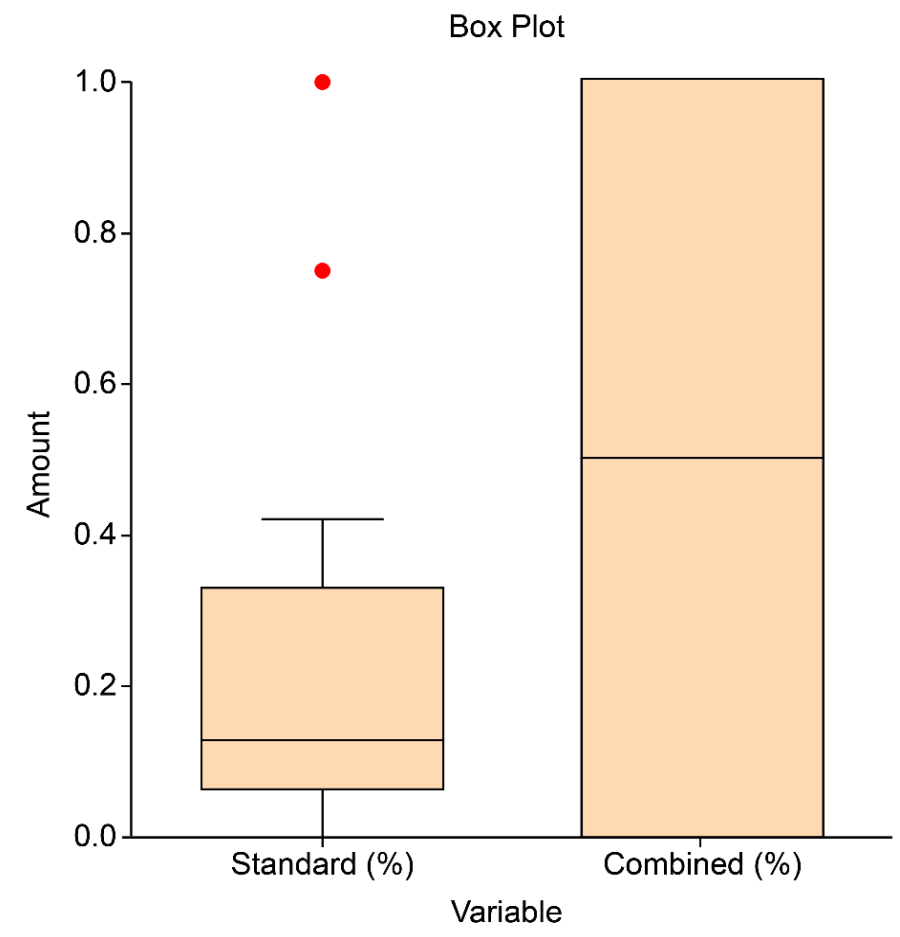

Figure 5. Detection rate of both biopsy techniques. The combined biopsy technique yielded higher detection
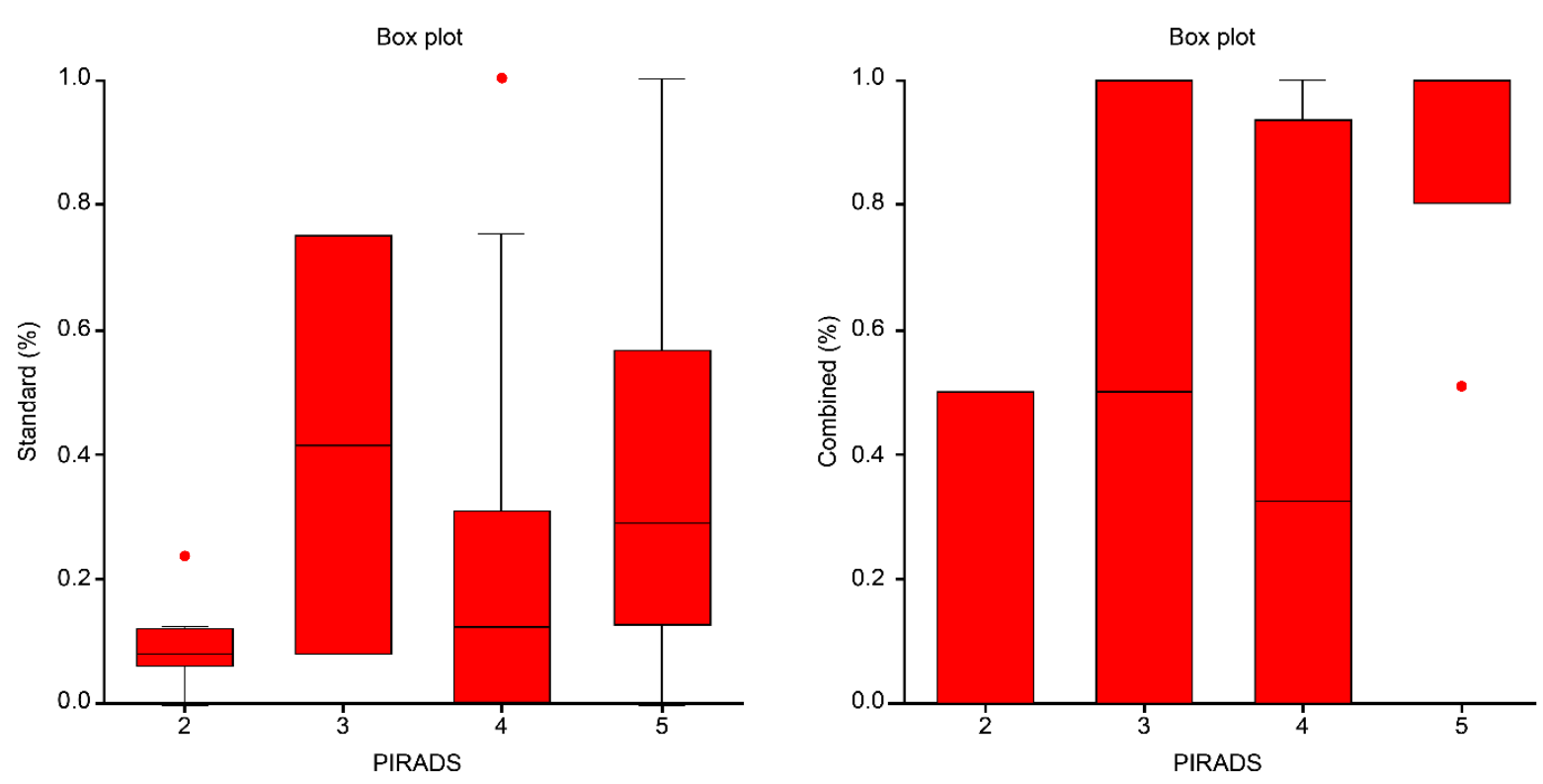

Figure 6. Both diagrams show the proportion between the standard and combined biopsy techniques on PIRADS results

In the case of 60 patients who underwent only mpMRI, we contacted $38(63 \%)$ men to ask what their physician recommended after MRI. The remaining 22 (37\%) patients were not available or did not provide any information.

Of these patients, $58 \%(n=22)$ had a PIRADS score of 2 and follow up was recommended by their physician, three patients $(13 \%(n=5))$ with a PIRADS score of 3 underwent a $\mathrm{PPBx}$ resulting in PCa, and two patients remain under surveillance. Two patients with a PIRADS score of $4(21 \% / \mathrm{n}=8)$ underwent $\mathrm{fPBx}$ with positive results, and three patients had negative standard biopsies with their urologists. Three men were under surveillance at the time we contacted them. The three patients with PIRADS scores of $5(8 \% / n=3)$ obtained a fPBx showing prostate cancer.

\section{Discussion}

Our study reveals that men with mpMRI results $\bigotimes$ PIRADS 3 should undergo a standard + target biopsy.

A repeat prostate biopsy is recommended for patients with initially negative biopsies but a lingering suspicion of PCa, i.e., elevated PSA levels, a suspect DRE, or ASAP or HGPIN in prior biopsies [20]. In 
addition to the quality of the initial biopsies, another important factor affecting the rate of false-negative results is the quality of the samples obtained in the extended initial biopsies, which yield a false-negative rate of up to $30 \%$. Newer imaging modalities are more accurate in visualizing and detecting clinically significant $\mathrm{PCa}$, facilitating the precise sampling of ROIs [2,21].

We examined the role of mpMRI and $\mathrm{fPBx}$ in patients undergoing at least their second biopsy and in young patients (median age 52,9 years) with a suspicion of PCa based on the combined biopsy techniques as a part of the first workup. To evaluate the MRI results of these patients, we included a large number of patients who underwent prostate mpMRI but did not have a biopsy. The patients underwent MRI alone according to the discretion of their treating urologists. In this cohort, most men (60\%) had MRIs with a PIRADS score of 2, while the remaining $40 \%$ had PIRADS scores of 3-5. The MRI results were analyzed by two uroradiologists. The acquired information about these patients underlines the necessity that a $\mathrm{PBBx}$ should be required in cases beginning at PIRADS 3. Patients with PIRADS 4 still undergoing surveillance or standard biopsies, should avoid standard biopsies which are significantly inferior to $\mathrm{PPBx}$.

However, $24 \%$ of patients with a PIRADS score of $\leq 2$ were diagnosed with significant PCa [22]. Additionally, biopsy remains the method of choice to verify or exclude any prostate malignancies.

A pre-evaluation rationalizing the use of MRI prior to biopsy could be an aim in PCa diagnosis to limit biopsies, but it is not currently recommended [23]. The EAU guidelines recommend a repeat biopsy using mpMRI in cases of persisting clinical suspicion of PCa [24]. Although the PROMIS study showed that $25 \%$ of their patients underwent mpMRI as a triage test before conducting the first prostate biopsy [15,25], the usefulness of mpMRI alone is questionable if the biopsy is excluded in an a-priori decision. Moreover, the combined method of mpMRI plus fPBx should be recommended for patients who must undergo a repeat prostate biopsy; furthermore, for special cases (i.e., young patients and a high suspicion of $\mathrm{PCa}$ ), mpMRI can provide additional information for detecting PCa as soon as possible.

In our study, the number of positive specimens was significantly higher in the $\mathrm{PBBx}$ cohort than in the standard biopsy cohort $(81 / 161$ vs. 101/468; $\mathrm{p}<0.0001$ ) among those with confirmed PCa. In the current study, any up- or downgrading of the imaging and definitive pathological results after prostatectomy were not evaluated, unlike in a previous study [23]. The mean GS of the standard (6.9; SD, 1.0) and combined biopsy techniques $(7.1 ; \mathrm{SD}, 0.9)$ is inadequate for drawing conclusive results. The number of clinically significant lesions confirmed via biopsy was lower than those confirmed via mpMRI. A total of 20 (60.6\%) patients with verified PCa had a GS of $\otimes 7$, while 13 (39.4\%) had a GS of 6 , providing evidence that mpMRI tends to detect higher risk disease. The selected patients receiving $\mathrm{mpMRI}$ and $\mathrm{PPBx}$ for firstline diagnosis were young (aged between 46 and 61 years, median 52,9 years) and in excellent physical condition. Patients who knew the facts and advantages of mpMRI and $\mathrm{PBx}$ underwent the combined biopsy technique for first-line diagnosis. According to the results, nearly $70 \%$ of patients with first-line biopsy had $\mathrm{PCa}$, demonstrating that patients should undergo mpMRI and PPBx for early detection of detect PCa and not only for rebiopsies.

The detection rate and accuracy of both biopsy methods based on the PIRADS findings present better results with the combined biopsy technique than with the standard biopsy technique (Figure 6). For PIRADS 2, we found better results with the standard biopsy technique, but it should be noted that this finding was because of the total number of cases. Of course, there is the question of whether PIRADS 2 should be biopsied at all.

Combining mpMRI with $\mathrm{PBBx}$ may reduce the overdiagnosis of clinically insignificant cancers, while improving the detection rate of clinically significant cancers compared with the standard TRUS biopsy [15]. In general, $\mathrm{fPBx}$ has a similar performance to MRI-targeted biopsy in terms of detecting PCa and other clinically significant cancers [1], although $\mathrm{PPBx}$ has the advantage of being less complex. Additionally, targeted cores contain more tumors than systematic cores and improve risk stratification and therapy planning [26,27]. However, this modality should be improved to reduce the number of collected biopsy cores, avoiding general anesthesia and the need for an MRI-guided biopsy to accurately determine the positive biopsies of significant PCa. Imaging techniques continue to play an prominent role in whether detecting clinically significant $\mathrm{PCa}$ is important in active surveillance and enable the possibility for focal therapy $[28,29]$. Finally, the combination of MRI and ultrasound, including fusion biopsy, increases the detection rate of PCa, particularly clinically significant PCa. Standard biopsy alone has a lower detection rate than $\mathrm{PBX}$. Thus, mpMRI is definitely a useful supporting modality in detecting PCa for first-line diagnosis, particularly in special cases such as in this study, and offers the possibility of early PCa detection.

mpMRI alone is insufficient as a diagnostic modality for patients with suspected $\mathrm{PCa}$ and even for determining patient management, and for best results and best patient care, a combination of all available methods should be performed [30].

\section{Conclusion}

The combination of MRI and ultrasound, including fusion biopsy, increases the detection rate of $\mathrm{PCa}$, particularly clinically significant PCa. Standard biopsy alone has a lower detection rate than $\mathrm{PPBx}$. Thus, mpMRI is definitely a useful supporting modality for detecting PCa as well as first-line diagnosis. Importantly, our findings indicate that the use of mpMRI alone is inadequate as a diagnostic modality for patients with suspected PCa. Especially used as single tool occasionally without consequences concerning PIRADS $\otimes 3$ results. In this case a fusion biopsy is strongly recommended.

\section{References}

1. Wegelin O, Van Melick HHE, Hooft L, Bosch JLHR, Reitsma HB, et al. (2017) Comparing three different techniques for magnetic resonance imaging-targeted prostate biopsies: a systematic review of in-bore versus magnetic resonance imaging-transrectal ultrasound fusion versus cognitive registration. Is there a preferred technique? Eur Urol 71: 517-531. [Crossref]

2. Ukimura O, Coleman JA, De la Taille A, Emberton M, Epstein JI, et al. (2013) Contemporary role of systematic prostate biopsies: indications, techniques, and implications for patient care. Eur Urol 63: 214-230. [Crossref]

3. Ukimura O, De Castro Abreu AL, Gill IS, Shoji S, Hung AJ, et al. (2013) Image visibility of cancer to enhance targeting precision and spatial mapping biopsy for focal therapy of prostate cancer. BJU Int 111: E354-E364. [Crossref]

4. Záleský M, Stejskal J, Adamcova V, Hrbáček J, Minarik I, et al. (2019) Use of Prostate Specific Antigen Density Combined with Multiparametric Magnetic Resonance Imaging Improves Triage for Prostate Biopsy. Urol Int 103: 33-40. [Crossref]

5. Heijmink SW, Van Moerkerk H, Kiemeney LA, Witjes JA, Frauscher F, et al. (2006) A comparison of the diagnostic performance of systematic versus ultrasound-guided biopsies of prostate cancer. Eur Radiol 16: 927-938. [Crossref]

6. Taira AV, Merrick GS, Galbreath RW, Andreini H, Taubenslag W, et al. (2010) Performance of transperineal template-guided mapping biopsy in detecting prostate cancer in the initial and repeat biopsy setting. Prostate Cancer Prostatic Dis 13: 71-77. [Crossref] 
7. Sonn GA, Chang E, Natarajan S, Margolis DJ, Macairan M, et al. (2014) Value of targeted prostate biopsy using magnetic resonance-ultrasound fusion in men with prior negative biopsy and elevated prostate-specific antigen. Eur Urol 65: 809-815. [Crossref]

8. Welch HG, Fisher ES, Gottlieb DJ, Barry MJ (2007) Detection of prostate cancer via biopsy in the medicare-SEER population during the PSA era. J Natl Cancer Inst 99: 1395-1400. [Crossref]

9. Hamann MF, Hamann C, Trettel A, Junemann KP, Naumann CM (2015) Computeraided transrectal ultrasound: does prostate histoscanning improve detection performance of prostate cancer in repeat biopsies? BMC Urol 15: 76. [Crossref]

10. Lunacek A, Simon J, Bernt R, Huber M, Plas E, et al. (2013) Increased rate of positive biopsies using a combination of MR-tomography, spectroscopy and diffusion-weighted magnetic resonance imaging prior to prostate biopsies in patients with persistent elevated prostate-specific antigen values: a retrospective analysis. Urol Ann 5: 76-80. [Crossref]

11. Chun FK, Briganti A, Shariat SF, Graefen M, Montorsi F, et al. (2006) Significant upgrading affects a third of men diagnosed with prostate cancer: predictive nomogram and internal validation. BJU Int 98: 329-334. [Crossref]

12. Mazaheri Y, Akin O, Hricak H (2017) Dynamic contrast-enhanced magnetic resonance imaging of prostate cancer: a review of current methods and applications. World $J$ Radiol 9: 416-425. [Crossref]

13. Descotes JL (2019) Diagnosis of prostate cancer. Asian J Urol 6: 129-136. [Crossref]

14. Draulans C, Everaerts W, Isebaert S, Gevaert T, Oyen R, et al. (2019) Impact of MRI on prostate cancer staging and EAU risk classification. Urology 130: 113-119. [Crossref]

15. Ahmed HU, El-Shater Bosaily A, Brown LC, Gabe R, Kaplan R, et al. (2017) Diagnostic accuracy of multi-parametric MRI and TRUS biopsy in prostate cancer (PROMIS): a paired validating confirmatory study. Lancet 389: 815-822. [Crossref]

16. Tong A, Flemming K, McInnes E, Oliver S, Craig J (2012) Enhancing transparency in reporting the synthesis of qualitative research: ENTREQ. BMC Med Res Methodol 12: 181. [Crossref]

17. Weinreb JC, Barentsz JO, Choyke PL, et al. (2016) PI-RADS prostate imaging reporting and data system: 2015, version 2. Eur Urol 69: 16-40. [Crossref]

18. Tran GN, Leapman MS, Nguyen HG, Cowan JE, Shinohara K, et al. (2017) Magnetic resonance imaging-ultrasound fusion biopsy during prostate cancer active surveillance. Eur Urol 72: 275-281. [Crossref]

19. Lunacek A, Mrstik C, Simon J, Frauscher F, Schwentner C, et al. (2014) Combination of lidocaine suppository and periprostatic nerve block during transrectal prostate biopsy: a prospective randomized trial. Int J Urol 21: 1126-30. [Crossref]
20. Heidenreich A, Bellmunt J, Bolla M, Joniau S, Mason M, et al. (2011) EAU guidelines on prostate cancer. Part 1: screening, diagnosis, and treatment of clinically localised disease. Eur Urol 59: 61-71. [Crossref]

21. Hambrock T, Somford DM, Hoeks C, Bouwense SA, Huisman H, et al. (2010) Magnetic resonance imaging guided prostate biopsy in men with repeat negative biopsies and increased prostate specific antigen. J Urol 183: 520-527. [Crossref]

22. Sonn GA, Fan RE, Ghanouni P, Wang NN, Brooks JD, et al. (2017) Prostate magnetic resonance imaging interpretation varies substantially across radiologists. Eur Urol Focus 5: 592-599. [Crossref]

23. Moldovan PC, Van den Broeck T, Sylvester R, Marconi L, Bellmunt J, et al. (2017) What is the negative predictive value of multiparametric magnetic resonance imaging in excluding prostate cancer at biopsy? A systematic review and meta-analysis from the European association of urology prostate cancer guidelines panel. Eur Urol 72: 250-266. [Crossref]

24. Mottet N, Bellmunt J, Bolla M, Briers E, Cumberbatch MG, et al. EAU - ESTRO - ESUR - SIOG guidelines on prostate cancer 5. Diagnostic evaluation 5.2.4.3. Guidelines for imaging.16-29.

25. Siddiqui MM, Rais-Bahrami S, Truong H, Stamatakis L, Vourganti S, et al. (2013) Magnetic resonance imaging/ultrasound-fusion biopsy significantly upgrades prostate cancer versus systematic 12-core transrectal ultrasound biopsy. Eur Urol 64: 713-719. [Crossref]

26. Schlenker B, Apfelbeck M, Buchner A, Stief C, Clevert DA (2018) MRI-TRUS fusion biopsy of the prostate: Quality of image fusion in a clinical setting. Clin Hemorheol Microcirc 70: 433-440. [Crossref]

27. Abdi H, Pourmalek F, Zargar H, Walshe T, Harris AC, et al. (2015) Multiparametric magnetic resonance imaging enhances detection of significant tumor in patients on active surveillance for prostate cancer. Urology 85: 423-428. [Crossref]

28. Tavolaro S, Mozer P, Roupret M, Comperat E, Rozet F, et al. Transition zone and anterior stromal prostate cancers: evaluation of discriminant location criteria using multiparametric fusion-guided biopsy. Diagn Interv Imaging 99: 403-411. [Crossref]

29. Algohary A, Viswanath S, Shiradkar R, Ghose S, Pahwa S, et al. (2018) Radiomic features on MRI enable risk categorization of prostate cancer patients on active surveillance: preliminary findings. J Magn Reson Imaging 48: 818-828. [Crossref]

30. Rouviere O, Moldovan PC (2019) The current role of prostate multiparametric magnetic resonance imaging. Asian J Urol 6: 137-145.

Copyright: (C2019 Lunacek A. This is an open-access article distributed under the terms of the Creative Commons Attribution License, which permits unrestricted use, distribution, and reproduction in any medium, provided the original author and source are credited. 